\title{
Hot nuclear matter in the quark meson coupling model
}

\author{
P. K. Panda, ${ }^{1}$ A. Mishra, ${ }^{1, *}$ J. M. Eisenberg, ${ }^{1,2, \dagger}$ and W. Greiner ${ }^{1}$ \\ ${ }^{1}$ Institut für Theoretische Physik, J. W. Goethe Universität, Robert Mayer-Straße 10, Postfach 1119 32, \\ D-60054 Frankfurt/Main, Germany \\ ${ }^{2}$ School of Physics and Astronomy, Raymond and Beverly Sackler Faculty of Exact Sciences, Tel Aviv University,
} 69978, Tel Aviv, Israel

(Received 27 May 1997)

\begin{abstract}
We study here hot nuclear matter in the quark meson coupling model which incorporates explicitly quark degrees of freedom, with quarks coupled to scalar and vector mesons. The equation of state of nuclear matter including the composite nature of the nucleons is calculated at finite temperatures. The calculations are done taking into account the medium-dependent bag constant. Nucleon properties at finite temperatures as calculated here are found to be appreciably different from the value at $T=0$. [S0556-2813(97)03512-7]
\end{abstract}

PACS number(s): 21.65.+f, 24.85.+p, 12.39.Ba, 12.38.Lg

\section{INTRODUCTION}

Quantum hadrodynamics (QHD) is a model for the nuclear many-body problem [1-3] describing nucleons interacting with scalar $(\sigma)$ and vector $(\omega)$ mesons. In the mean field approximation for the meson fields, it has been shown that the ground-state properties of nuclear matter may be fitted by adjusting the scalar and vector couplings [4]. This meson field theory has quite successfully described the properties of nuclear matter as well as finite nuclei using hadronic degrees of freedom. The vacuum polarization corrections arising from the nucleon fields [5] as well as the meson fields $[6,7]$ have also been considered to study nuclear matter. This leads to a softer equation of state, giving a lower value of the incompressibility than would be reached without quantum effects [7].

While descriptions of the nuclear phenomena have been efficiently formulated using hadronic degrees of freedom as in QHD, there have been interesting observations such as the EMC effect [8] which reveal the medium modification of the internal structure of the nucleon. This necessitates models that incorporate quark-gluon degrees of freedom respecting the established models based on the hadronic degrees of freedom. The quark meson coupling (QMC) model [9] is a simple extension of QHD incorporating the quark degrees of freedom. This model describes nuclear matter with nucleons as nonoverlapping MIT bags and the quarks inside them couple to scalar and vector mesons. Though it is simple and attractive, this model predicts smaller values for the scalar and vector potentials leading to a weaker nucleon spin-orbit force in finite nuclei. The understanding of the large and canceling values for the potentials, which has been central to the success of nuclear phenomenology, has been attempted recently through an analysis of a finite-density QCD sumrule calculations [10] and is related to medium effects. This is suggestive of including a medium-dependent bag constant in the QMC model $[11,12]$. We intend to study here nuclear matter using this variation of the QMC model at finite tem-

\footnotetext{
*Electronic address: mishra@th.physik.uni-frankfurt.de

${ }^{\dagger}$ Electronic address: judah@giulio.tau.ac.il
}

peratures taking the medium-dependence of the bag parameters into account.

We organize the paper as follows: In Sec. II, we briefly recapitulate the QMC model for nuclear matter at zero temperature [11], and in Sec. III we generalize it to finite temperature with a specific ansatz for the density-dependent bag constant. Finally, in Sec. IV, we summarize the results as obtained in this model, discuss its limitations, and present an outlook.

\section{QMC MODEL FOR NUCLEAR MATTER}

We here briefly recapitulate the QMC model for nuclear matter at zero temperature [11]. In this model, the nucleon in nuclear matter is assumed to be described by a static MIT bag in which quarks interact with the scalar $(\sigma)$ and the vector $(\omega)$ fields, which are treated as classical in a mean field approximation.

The quark field $\psi_{q}(\vec{r}, t)$ inside the bag then satisfies the equation

$$
\left[i \gamma^{\mu} \partial_{\mu}-\left(m_{q}^{0}-g_{\sigma}^{q} \sigma\right)-g_{\omega}^{q} \omega \gamma^{0}\right] \psi_{q}(\vec{r}, t)=0,
$$

where $m_{q}^{0}$ is the current quark mass and $g_{\sigma}^{q}$ and $g_{\omega}^{q}$ are the quark couplings with the $\sigma$ and $\omega$ mesons.

The normalized ground state for a quark (in an $s$ state) in the bag is given as

$$
\psi_{q}(\vec{r}, t)=N \exp \left(-i \frac{\epsilon_{q} t}{R}\right)\left(\begin{array}{c}
j_{0}(x r / R) \\
i \beta_{q} \vec{\sigma} \cdot \hat{r} j_{1}(x r / R)
\end{array}\right) \frac{\chi_{q}}{\sqrt{4 \pi}} .
$$

Then the single particle quark energy in units of $R^{-1}$ is

$$
\epsilon_{q}=\Omega_{q}+g_{\omega}^{q} \omega R, \quad \beta_{q}=\sqrt{\frac{\Omega_{q}-R m_{q}^{*}}{\Omega_{q}+R m_{q}^{*}}},
$$

with $\Omega_{q}=\left(x^{2}+R^{2} m_{q}^{* 2}\right)^{1 / 2} ; m_{q}^{*}=m_{q}^{0}-g_{\sigma}^{q} \sigma$ is the effective quark mass, $R$ is the bag radius, $\chi_{q}$ is the quark spinor, and $N$ is the normalization factor.

The boundary condition at the bag surface is given by

$$
i \gamma \cdot n \psi_{q}=\psi_{q}
$$


This for the ground state reduces to

$$
j_{0}(x)=\beta_{q} j_{1}(x)
$$

which determines the dimensionless quark momentum $x$. The energy of the nucleon bag is

$$
E_{\mathrm{bag}}=3 \frac{\Omega_{q}}{R}-\frac{Z}{R}+\frac{4}{3} \pi R^{3} B
$$

where $Z$ is a parameter accounting for the zero-point energy and $B$ is the bag constant. After subtracting spurious centerof-mass motion inside the bag, the effective mass of the nucleon bag at rest is given as [11]

$$
M_{N}^{*}=\sqrt{E_{\mathrm{bag}}^{2}-\left\langle p_{\mathrm{c} . \mathrm{m} .}^{2}\right\rangle},
$$

with $\left\langle p_{\text {c.m. }}^{2}\right\rangle=\Sigma_{q}\left\langle p_{q}^{2}\right\rangle \equiv 3(x / R)^{2}$. The bag radius $R$ is then obtained through

$$
\frac{\partial M_{N}^{*}}{\partial R}=0
$$

The total energy density of nuclear matter at baryon density $\rho_{B}$ is given in the usual form as

$$
\epsilon=\frac{\gamma}{(2 \pi)^{3}} \int^{k_{F}} d^{3} k \sqrt{k^{2}+M_{N}^{* 2}}+\frac{g_{\omega}^{2} \rho_{B}^{2}}{2 m_{\omega}^{2}}+\frac{1}{2} m_{\sigma}^{2} \sigma^{2},
$$

where $\gamma=4$ is the spin-isospin degeneracy factor for nuclear matter. The vector mean field $\omega$ is determined through

$$
\omega=\frac{g_{\omega} \rho_{B}}{m_{\omega}^{2}},
$$

where $g_{\omega}=3 g_{\omega}^{q}$. Finally, the scalar mean field $\sigma$ is fixed by

$$
\frac{\partial \epsilon}{\partial \sigma}=0
$$

The scalar and vector couplings $g_{\sigma}^{q}$ and $g_{\omega}^{q}$ are fitted to the saturation density and binding energy for nuclear matter. For a given baryon density, $x, R$, and $\sigma$ are calculated from the Eqs. (5), (8), and (11), respectively.

In the simple QMC model $[9,13]$, the bag constant $B$ is taken as $B_{0}$ corresponding to the bag parameter for a free nucleon. The medium effects are taken into account in the modified QMC model [11]. To include the finite-temperature effects for nuclear matter, we choose a specific ansatz for the medium-dependent bag parameter [11],

$$
B=B_{0} \exp \left(-\frac{4 g_{\sigma}^{B} \sigma}{M_{N}}\right)
$$

with $g_{\sigma}^{B}$ as an additional parameter. For a fixed set of bag parameters as taken in the Ref. [11], we now generalize the modified QMC model to study nuclear matter at finite temperatures.

\section{NUCLEAR MATTER AT FINITE TEMPERATURES}

At finite temperatures, the quarks inside the bag can be thermally excited to higher angular momentum states. For simplicity, we shall still assume the bag describing the nucleon to be spherical with radius $R$, which is now temperature dependent. This may not be a valid assumption at high temperatures as there should be corrections arising due to deformation of the nucleon bag. However, we believe that those corrections will not be significant enough so as to change the qualitative results of the present calculations.

Here the single-particle quark and antiquark energies in units of $R^{-1}$ are given as

$$
\epsilon_{ \pm}^{n \kappa}=\Omega^{n \kappa} \pm g_{\omega}^{q} \omega R
$$

where

$$
\Omega^{n \kappa}=\left(x_{n \kappa}^{2}+R^{2} m_{q}^{* 2}\right)^{1 / 2}
$$

$m_{q}^{*}=m_{q}^{0}-g_{\sigma}^{q} \sigma$ is the effective quark mass. The boundary condition at the bag surface, given by

$$
i \gamma \cdot n \psi_{q}^{n \kappa}=\psi_{q}^{n \kappa}
$$

now determines the quark momentum $x_{n \kappa}$ in the state characterized by specific values of $n$ and $\kappa$. The total energy from the quarks and antiquarks is

$$
E_{\mathrm{tot}}=3 \sum_{n, \kappa} \frac{\Omega^{n \kappa}}{R}\left[\frac{1}{e^{\left(\epsilon_{+}^{n \kappa} / R-\mu_{q}\right) / T}+1}+\frac{1}{e^{\left(\epsilon_{-}^{n \kappa} / R+\mu_{q}\right) / T}+1}\right],
$$

and the bag energy now becomes

$$
E_{\mathrm{bag}}=E_{\mathrm{tot}}-\frac{Z}{R}+\frac{4 \pi}{3} R^{3} B
$$

which reduces to Eq. (6) at zero temperature. The spurious center-of-mass motion in the bag is subtracted to obtain the effective nucleon mass

$$
M_{N}^{*}=\left(E_{\mathrm{bag}}^{2}-\left\langle p_{\mathrm{c} . \mathrm{m} .}^{2}\right\rangle\right)^{1 / 2}, \quad\left\langle p_{\mathrm{c} . \mathrm{m} .}^{2}\right\rangle=\frac{\left\langle x^{2}\right\rangle}{R^{2}},
$$

where

$$
\left\langle x^{2}\right\rangle=3 \sum_{n, \kappa} x_{n \kappa}^{2}\left[\frac{1}{e^{\left(\epsilon_{+}^{n \kappa} / R-\mu_{q}\right) / T}+1}+\frac{1}{e^{\left(\epsilon_{-}^{n \kappa} / R+\mu_{q}\right) / T}+1}\right]
$$

is the spurious center-of-mass average momentum squared [11]. This is here written in terms of the sum of the quark and the antiquark distributions since the center-of-mass motion does not distinguish between a quark and an antiquark.

The quark chemical potential $\mu_{q}$, assuming that there are three quarks in the nucleon bag, is determined through

$$
n_{q}=3=3 \sum_{n, \kappa}\left[\frac{1}{e^{\left(\epsilon_{+}^{n \kappa / R}-\mu_{q}\right) / T}+1}-\frac{1}{e^{\left(\epsilon_{-}^{n \kappa} / R+\mu_{q}\right) / T}+1}\right] .
$$




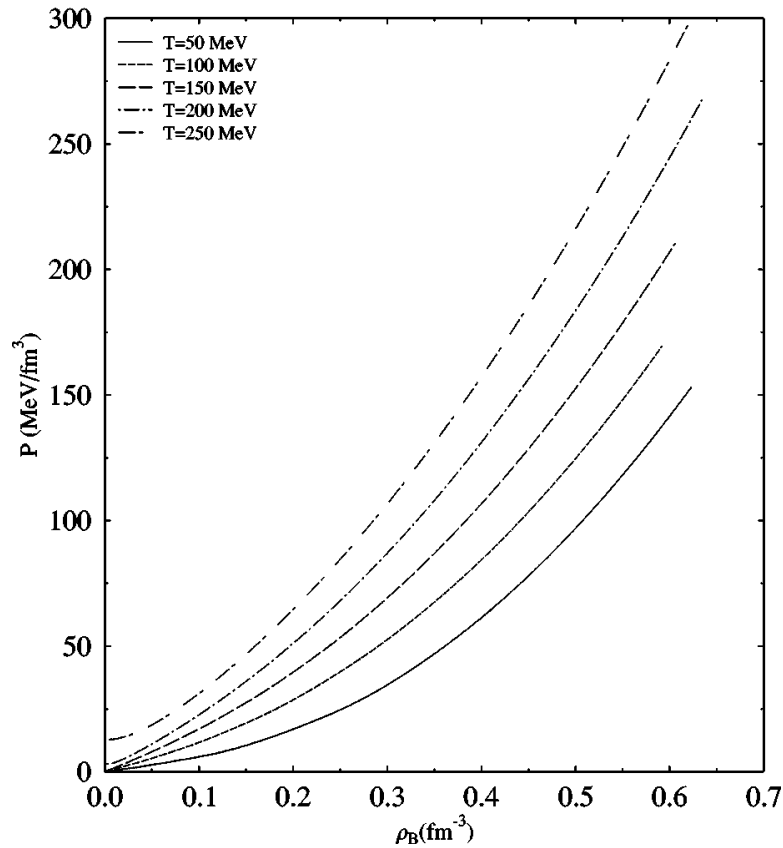

FIG. 1. The pressure for nuclear matter as a function of the baryon density $\rho_{B}$ for various values of temperature showing an increase with temperature.

The temperature-dependent bag radius $R$ is obtained in the same way as was done at zero temperature using Eq. (8).

The total energy density at finite temperature $T$ and at finite baryon density $\rho_{B}$ is [14]

$$
\epsilon=\frac{\gamma}{(2 \pi)^{3}} \int d^{3} k \sqrt{\vec{k}+M_{N}^{* 2}}\left(f_{B}+\bar{f}_{B}\right)+\frac{g_{\omega}^{2}}{2 m_{\omega}^{2}} \rho_{B}^{2}+\frac{1}{2} m_{\sigma}^{2} \sigma^{2},
$$

where $f_{B}$ and $\bar{f}_{B}$ are the thermal distribution functions for the baryons and antibaryons,

$$
f_{B}=\frac{1}{e^{\left(\epsilon^{*}(\vec{k})-\mu_{B}^{*}\right) / T}+1} \quad \text { and } \bar{f}_{B}=\frac{1}{e^{\left(\epsilon^{*}(\vec{k})+\mu_{B}^{*}\right) / T}+1}
$$

with $\epsilon^{*}(\vec{k})=\left(\vec{k}^{2}+M_{N}^{* 2}\right)^{1 / 2}$ the effective nucleon energy and $\mu_{B}^{*}=\mu_{B}-g_{\omega} \omega$ the effective baryon chemical potential.

The thermodynamic grand potential is

$$
\begin{aligned}
\Omega= & \epsilon-T S-\mu_{B} \rho_{B}=\frac{\gamma}{(2 \pi)^{3}} \int d^{3} k \epsilon^{*}(k)\left(f_{B}+\bar{f}_{B}\right)+\frac{g_{\omega}^{2}}{2 m_{\omega}^{2}} \rho_{B}^{2} \\
& +\frac{1}{2} m_{\sigma}^{2} \sigma^{2}-T S-\mu_{B} \rho_{B},
\end{aligned}
$$

with the entropy density

$$
\begin{aligned}
S= & -\frac{\gamma}{(2 \pi)^{3}} \int d^{3} k\left[f_{B} \ln f_{B}+\left(1-f_{B}\right) \ln \left(1-f_{B}\right)+\bar{f}_{B} \ln \bar{f}_{B}\right. \\
& \left.+\left(1-\overline{f_{B}}\right) \ln \left(1-\overline{f_{B}}\right)\right]
\end{aligned}
$$

and the baryon density

$$
\rho_{B}=\frac{\gamma}{(2 \pi)^{3}} \int d^{3} k\left(f_{B}-\bar{f}_{B}\right) .
$$

The pressure is the negative of $\Omega$, and, after integration by parts, reduces to the familiar expression [15]

$$
P=\frac{\gamma}{3} \frac{1}{(2 \pi)^{3}} \int d^{3} k \frac{\vec{k}^{2}}{\epsilon^{*}(k)}\left(f_{B}+\bar{f}_{B}\right)+\frac{g_{\omega}^{2}}{2 m_{\omega}^{2}} \rho_{B}^{2}-\frac{1}{2} m_{\sigma}^{2} \sigma^{2} .
$$

At finite temperatures and for a given $\mu_{B}$, the effective nucleon mass is known for given values of the meson fields once the bag radius $R$ and the quark chemical potential $\mu_{q}$ are calculated by using Eqs. (8) and (20), respectively. The mean field $\sigma$ is determined through the minimization of the thermodynamic potential and using the self-consistency condition

$$
\omega=\frac{g_{\omega}}{m_{\omega}^{2}} \frac{\gamma}{(2 \pi)^{3}} \int d^{3} k\left(f_{B}-\bar{f}_{B}\right) .
$$

\section{RESULTS AND DISCUSSIONS}

We now proceed with the finite-temperature calculations for nuclear matter. As already mentioned, we assume the density-dependent bag constant to be of the form given by Eq. (12) with $g_{\sigma}^{B}$ as a parameter. Further, we take the bag parameters for the free nucleon as $B_{0}^{1 / 4}=188.1 \mathrm{MeV}$ and $Z$ $=2.03[11]$ and the current quark mass $m_{q}^{0}$ as zero. For $g_{\sigma}^{q}$ $=1$, the values of the vector meson coupling and the parameter $g_{\sigma}^{B}$, as fitted from the saturation properties of nuclear matter [11], are given as $g_{\omega}^{2} / 4 \pi=5.24$ and $\left(g_{\sigma}^{B}\right)^{2} / 4 \pi=3.69$.

For specific values of the temperature and $\mu_{B}$, the thermodynamic potential is given in terms of the effective nucleon mass which depends on the bag radius $R$, the quark chemical potential $\mu_{q}$, and the mean fields $\sigma$ and $\omega$. For given values of $\sigma$ and $\omega$, the bag radius and the quark chemical potential are determined using the conditions (8) and (20), respectively. We then determine the value of $\sigma$ by minimizing the thermodynamic potential $\Omega$, with $\omega$ determined from the self-consistency condition of Eq. (27).

For given values of the temperature and $\mu_{B}$, we calculate the different thermodynamic quantities along with the bag properties, which are now temperature dependent. In Fig. 1, we plot the pressure as a function of the baryon density $\rho_{B}$ for different values of the temperature. For a given $\rho_{B}$, pressure has the usual trend of increasing with temperature [14]. The pressure for $\rho_{B}=0$ becomes nonzero at and above a temperature of $200 \mathrm{MeV}$. This has contributions arising from the thermal distribution functions for the baryons and antibaryons as well as from a nonzero value for the sigma field. The scalar sigma field attaining a nonzero value was also observed for nuclear matter in the Walecka model [14], which had led to a sharp fall in the effective nucleon mass between temperatures of $150 \mathrm{MeV}$ and $200 \mathrm{MeV}$. This rapid fall of $M_{N}^{*}$ with increasing temperature resembles a phase transition when the system becomes a dilute gas of baryons in a sea of baryon-antibaryon pairs.

The density- and temperature-dependence of the baryon 


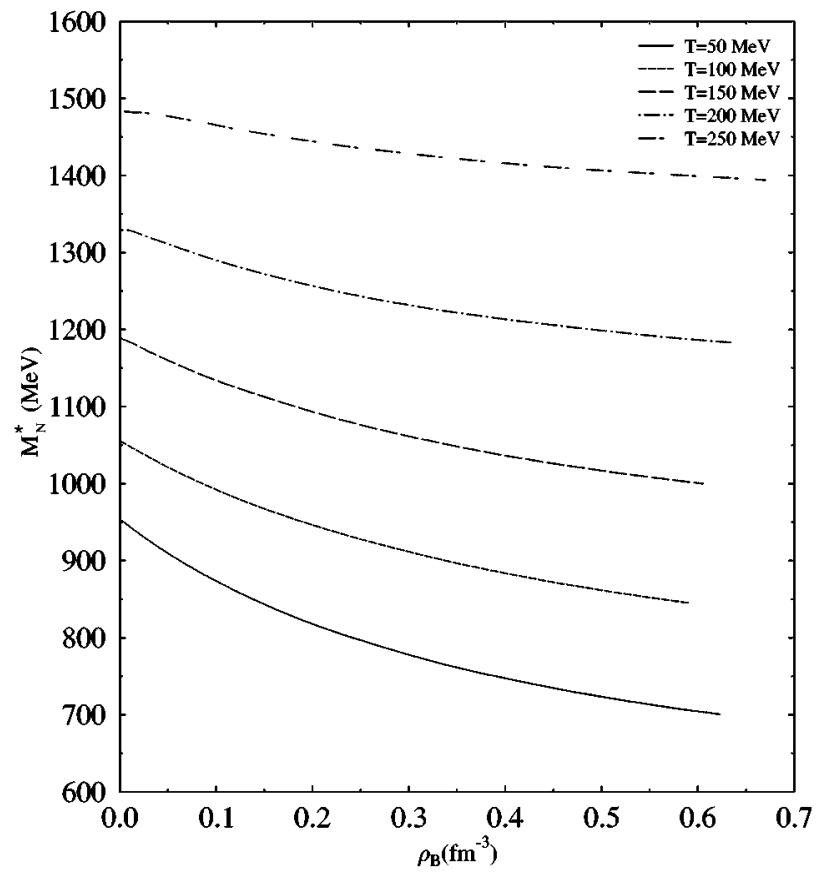

FIG. 2. The effective nucleon mass for nuclear matter as a function of the baryon density $\rho_{B}$ for different values of the temperature, which is seen to rise monotonically with temperature.

effective mass is shown in Fig. 2. The value of $M_{N}^{*}$ here increases monotonically with temperature. This is in contrast to the calculation of hot nuclear matter using Walecka model [14], where, as the temperature is increased, $M_{N}^{*}$ first rises and then falls rapidly for $T \simeq 200 \mathrm{MeV}$. We do not encounter any change in the trend here even up to a temperature of 250 $\mathrm{MeV}$, even though similar to earlier calculation, the sigma field becomes finite at a temperature of $200 \mathrm{MeV}$. The reason for this is that the sigma field here is not strong as in Ref. [14]. Also, there is a significant contribution to the effective mass arising from the thermal excitations of the quarks inside the nucleon bag. This adds to the mass of the nucleon whereas the nucleon mass decreases with increase in the value of the sigma field. The contribution of the former, which was absent in the Walecka model calculations [14], appears to dominate over the contribution from the nonzero value of $\sigma$ and the net effect is a rise of the effective nucleon mass in the present case. Hence, although in the earlier case [14] the effective mass is identical to the free nucleon mass at zero density and nonzero temperatures before the phase transition takes place, $M_{N}^{*}$ in our calculations has a higher value as compared to the nucleon mass in vacuum. A similar increase was also observed earlier for the $N$ and $\Delta$ masses for nonzero temperatures using the thermal skyrmions [16].

We then look at the dependence of the entropy density on the density and temperature as shown in Fig. 3. This has a nonzero value even at vanishing baryon density at and above a temperature of $200 \mathrm{MeV}$, with contributions from the nonzero value of the sigma field. A similar behavior was observed for the entropy density in the Walecka model calculations [14]. The change in the behavior of the entropy density in the earlier case was rather abrupt indicating a sharp phase transition. The present calculations show a softening of the phase transition.

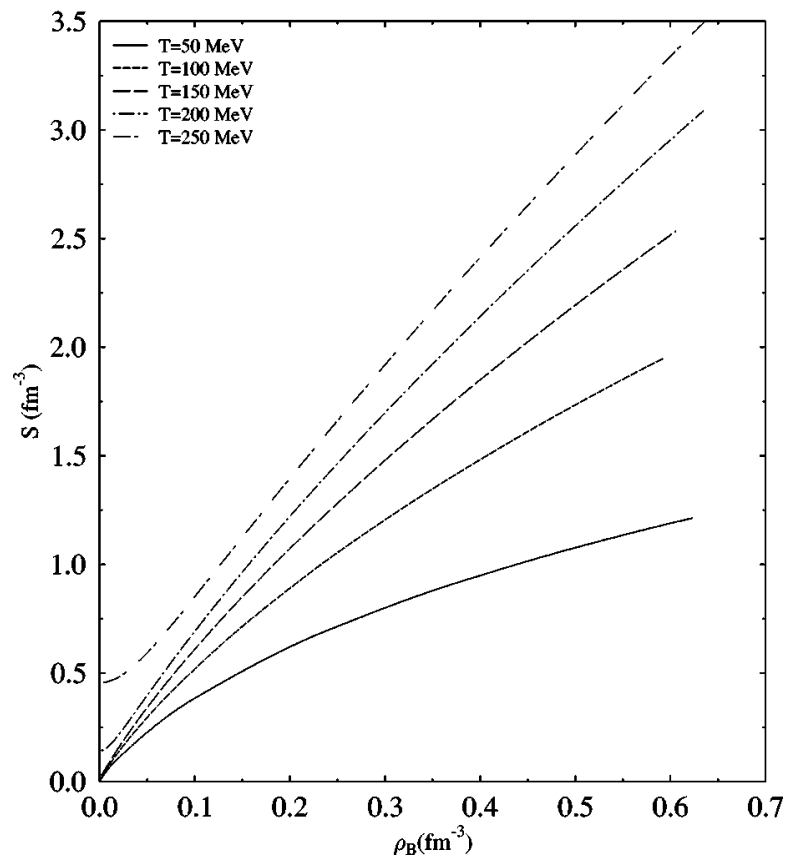

FIG. 3. The entropy density vs the density for various temperatures.

The mean field $\sigma$ as obtained through the minimization of the thermodynamic potential is plotted as a function of the baryon density for various temperatures in Fig. 4. The sigma field becomes nonzero at a temperature of $200 \mathrm{MeV}$, indicating a phase transition to a system of baryon-antibaryon pairs at very low densities as mentioned earlier. The value of $\sigma$ decreases with temperature at higher densities. A similar behavior was also observed earlier at temperatures before the phase transition took place [14], and had the natural consequence that the effective nucleon mass grows with temperature. However, at low densities and high temperatures, $\sigma$

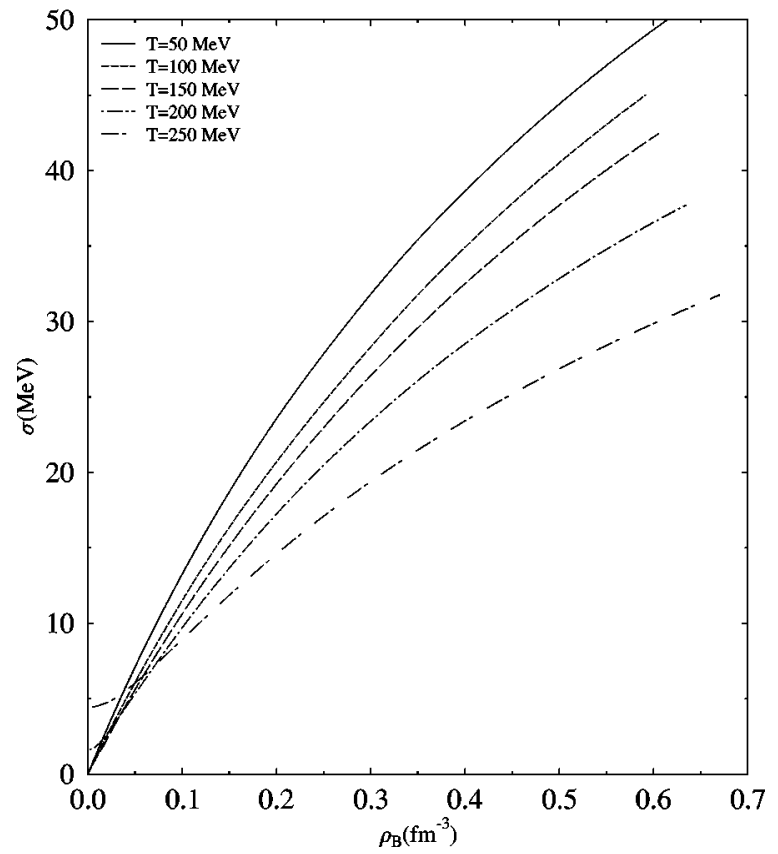

FIG. 4. The mean field $\sigma$ as a function of density for different temperatures. 


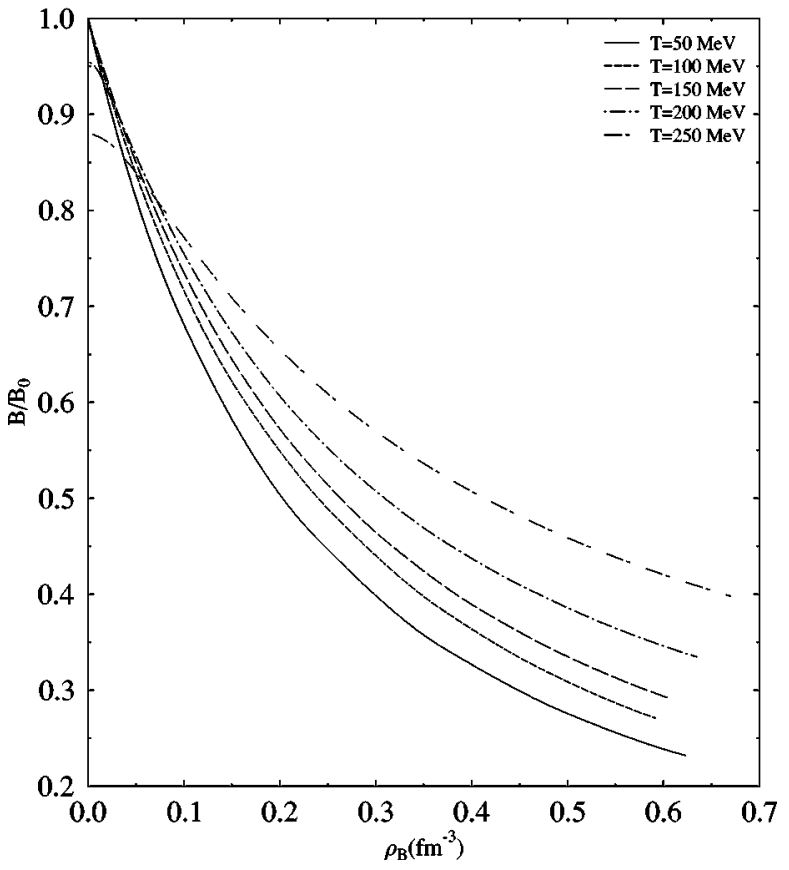

FIG. 5. The bag constant vs the density for different temperatures. It grows with temperature, except for very low densities and temperatures at and above $200 \mathrm{MeV}$.

increases with temperature thus reducing the effective mass in the earlier case. Even though the behavior of sigma is similar, the effective mass here is dominated by the thermal excitations of the quarks which leads to an increase in the effective mass with temperature.

We also look at some other properties of the nucleon bag at finite temperatures. The bag constant, as shown in Fig. 5, grows with temperature for densities greater than around $0.1 \mathrm{fm}^{-3}$. This is clear from the form of $B$, chosen as in Eq. (12), since the mean field $\sigma$ decreases with temperature. The bag constant at zero density is seen to be identical to the free nucleon bag constant for temperatures before the phase transition. However, at temperatures at and above $200 \mathrm{MeV}$, it starts decreasing with temperature. The temperature- and density-dependence of the bag radius, in units of the free nucleon radius $R_{0}=0.6$ fermis, is shown in Fig. 6. The nucleon bag in this model shrinks with increasing temperature. Such a difficulty of obtaining a baryon that swells with temperature was also encountered using the thermal skyrmion $[16,17]$. A possible solution for the difficulty was suggested in the earlier case $[16,17]$ whereby the thermal skyrmion exists in a bath of pions, which effectively increase its radius.

Let us discuss some limitations of the present model before summarizing the results. The quark meson coupling model is a naive generalization of quantum hadrodynamics to include the quark substructure of the nucleons, while the mesons are still regarded as structureless. At finite temperatures and densities, it is not quite clear which many body effects should be attributed to quarks and which to mesons, particularly so, near the phase transition. We have included here the thermal effects of the quarks alone. Further, the color degrees of freedom for the quarks, the deformations of the nucleon bag at finite temperatures and/or densities, are

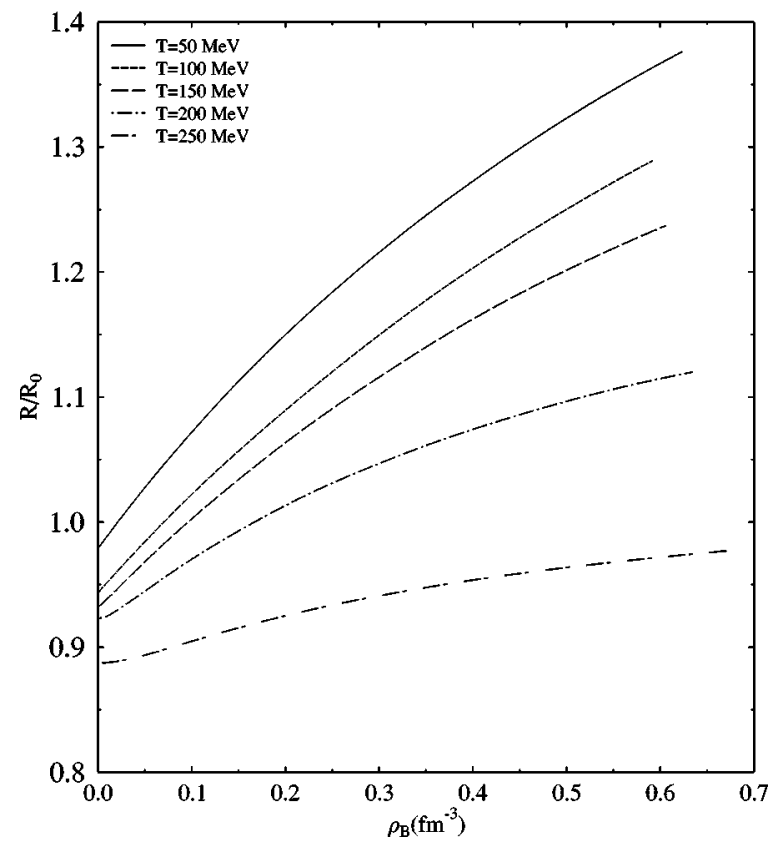

FIG. 6. The bag radius as a function of density at various temperatures; the nucleon bag size shrinks with temperature.

not taken into account in this simple description. However, within the perview of the QMC model accepted as is, we studied here nuclear matter at finite temperatures using the QMC model and taking into account the density dependence of the bag constant. The mean fields $\sigma$ and $\omega$ were determined through minimization of the thermodynamic potential and the temperature dependent bag radius was calculated by minimizing the effective mass of the nucleon bag. The scalar mean field $\sigma$ at zero density attains a nonzero value at a temperature of $200 \mathrm{MeV}$ similar to the Walecka model calculations for the nuclear matter [14], which is indicative of a phase transition to a system with baryon-antibaryon pairs. However, there is a softening in the phase transition here as compared to the earlier calculations. This is because the thermal contributions from the quarks, which were absent in Ref. [14], are dominant here and lead to a rise of the effective nucleon mass with temperature. The nucleon bag in the present calculation shrinks in size with increase in the temperature. The nucleon mass at finite temperatures and zero baryon density as calculated here is appreciably different from the nucleon mass in vacuum.

\section{ACKNOWLEDGMENTS}

The authors would like to acknowledge many useful discussions with Professor Henry Jaqaman, Professor B. K. Jennings, Dr. J. Reinhardt, Dr. M. Belkacem, Dr. S. Schramm, and Dr. H. Mishra. J.M.E. wishes to thank Professor Walter Greiner and the Institute for Theoretical Physics at the University of Frankfurt for their kind hospitality, and to acknowledge support from the Deutsche Forschungsgemeinschaft and the Ne'eman Chair in Theoretical Nuclear Physics at Tel Aviv University. A.M. and P.K.P. would like to thank the Alexander von Humboldt-Stiftung for financial support and the Institut für Theoretische Physik for warm hospitality. 
[1] B. D. Serot and J. D. Walecka, Adv. Nucl. Phys. 16, 1 (1986); B. D. Serot and J. D. Walecka, nucl-th/9701058, to appear in Int. J. Mod. Phys. E.

[2] P.-G. Reinhard, Rep. Prog. Phys. 52, 439 (1989).

[3] B. D. Serot, Rep. Prog. Phys. 55, 1855 (1992).

[4] J. D. Walecka, Ann. Phys. (N.Y.) 83, 491 (1974).

[5] S. A. Chin and J. D. Walecka, Phys. Lett. 52B, 24 (1974); S. A. Chin, Ann. Phys. (N.Y.) 108, 301 (1977); R. J. Perry, Phys. Lett. B 199, 489 (1987).

[6] W. R. Fox, Nucl. Phys. A495, 463 (1989); Ph.D. thesis, Indiana University, 1988.

[7] A. Mishra, P. K. Panda, S. Schramm, J. Reinhardt, and W. Greiner, Phys. Rev. C 56, 1380 (1997).

[8] EMC Collaboration, J. J. Aubert et al., Phys. Lett. 123B, 275 (1983).

[9] P. A. M. Guichon, Phys. Lett. B 200, 235 (1988); see also the recent review by K. Saito, nucl-th/9705019.

[10] T. D. Cohen, R. J. Furnstahl, D. K. Griegel, and X. Jin, Prog. Part. Nucl. Phys. 35, 221 (1995) and references therein; R. J. Furnstahl, X. Jin, and D. B. Leinweber, Phys. Lett. B 387, 253 (1996).

[11] X. Jin and B. K. Jennings, Phys. Rev. C 54, 1427 (1996); Phys. Lett. B 374, 13 (1996).

[12] X. Jin and B. K. Jennings, Phys. Rev. C 55, 1567 (1997).

[13] K. Saito and A. W. Thomas, Phys. Lett. B 327, 9 (1994).

[14] R. J. Furnstahl and B. D. Serot, Phys. Rev. C 41, 262 (1990).

[15] G. Kälbermann, J. M. Eisenberg, and B. Svetitsky, Nucl. Phys. A600, 436 (1996).

[16] J. Dey and J. M. Eisenberg, Phys. Lett. B 334, 290 (1994).

[17] M. A. Nowak and I. Zahed, Phys. Lett. B 230, 108 (1989); K. J. Eskola and K. Kajantie, Z. Phys. C 44, 347 (1989). 